\title{
Ethically we can no longer sit on the fence - a neuro- psychological perspective on the cerebrally hazardous contact sports
}

\author{
A B Shuttleworth-Edwards (PhD) \\ V J Whitefield (PhD) \\ Psychology Department, Rhodes University, Grahamstown
}

\begin{abstract}
Background and objective. The number of male and female contact sport participants is increasing worldwide. The aim of the review is to discuss the potential for deleterious sequelae of sports concussion (mild traumatic brain injury (MTBI)), and management thereof.

Discussion. Incidence of concussion in the field contact sports is high, not only for boxing, but also for soccer, football and especially rugby. An overview of studies investigating persistent deleterious cognitive and symptomatic outcome following cumulative sports MTBI suggests that individuals may be at risk for permanent neurological damage following participation in a contact sport. Established sequelae of traumatic brain injury (TBI) typically involving frontal systems include cognitive decline, behavioural changes such as diminished self-regulation and aggression, and increased risk for Alzheimer's disease. The presence of such consequences hidden within the context of the widely popularised contact sports, has societal implications that should be acknowledged. Compromised scholastic abilities and enhanced aggressive tendencies in association with sports MTBI are in need of further longitudinal research.
\end{abstract}

Conclusion. A comprehensive preventive approach to the management of MTBI in sport is advocated that includes professionally applied neuropsychological assessment as a crucial component. Future policy considerations are the introduction of mandatory informed consent for participation in a high-risk contact sport such as rugby, particularly at youth level, and financial provision for concussion management amongst economically disadvantaged populations.

\section{CORRESPONDENCE:}

A B Shuttleworth-Edwards

National Sports Concussion Initiative

Psychology Clinic

Rhodes University

Grahamstown

6139

Tel/Fax: 046-636 1296

E-mail: A.Edwards@ru.ac.za

\section{Introduction}

The purpose of this review is to highlight concerns regarding the consequences of mild traumatic brain injury (MBTI) (concussion) in the contact sports, including boxing, soccer, American football and rugby. Acute and chronic effects of concussion are discussed against a background of literature that sheds light on the incidence of concussion in these sports. Attention is drawn to individual implications, which in turn have societal ramifications in need of being acknowledged and researched. A way forward is proposed, including recommendations that apply specifically to the South African context.

\section{Increasing participation in contact sports}

The popularity of the traditionally male-dominated contact sports is growing, ${ }^{4}$ and of note is the increasing number of women who are participating in these sports. ${ }^{6}$ A media report suggests that in the early years of this decade more than 2000 women were already playing rugby football, and three women's Rugby Football Union World Cups had taken place involving 12 countries. ${ }^{5}$ In South Africa an official launch tournament was held in 2001 to promote Rugby Union for women, ${ }^{5}$ which is the rugby code followed in this country, as well as extensively in New Zealand, Australia and Britain. At around the same time in South Africa, legislation was drafted permitting women to participate in boxing, ${ }^{42}$ a move that follows a worldwide trend. In the past decade there has also been an active drive to extend sporting facilities to previously disadvantaged or racially precluded groups in South Africa. Together these factors mean that there are significantly increasing numbers of individuals participating in contact sport in this country, including both males and females, and individuals of all races from high to low socio-economic status. Accordingly, it appears important to evaluate the overall impact of exposure to head injury in these sports.

\section{Incidence of head injury in contact sport}

Incidence studies confirm that concussion is a common feature characterising the contact sports. Whilst it is self-evident that boxing is associated with repeated head insults, the degree to which this is an aspect of the field contact sports of football, soccer and rugby needs to be more fully acknowledged. Research on the incidence of concussion among American university-level football and soccer players during 
a single season reported figures of $34 \%$ and $46 \%$, respectively. ${ }^{11}$ Of these, $100 \%$ of the football players and $75 \%$ of the soccer players had suffered more than one concussion in the season. Due to the tackling manoeuvre in Rugby Union the rate of serious injuries in this sport is the highest among the rugby football sports; in particular there is a substantially higher rate of injury to the upper extremities, including the head and neck areas. ${ }^{44}$ The World Health Organization (WHO) task force review revealed a higher rate of concussion for Rugby Union than Rugby League, American football and soccer, being up to 8.0 per 1000 game hours, compared with an upper limit of 3.0, 3.3 and 1.3 per 1000 game hours respectively for the others. ${ }^{8}$ Studies on the contact sports in respect of gender generally indicate that females sustain around $50 \%$ fewer concussions than males. ${ }^{8}$

With specific reference to rugby, a New Zealand study ${ }^{49}$ found that Rugby Union accounted for the highest rate of sports-related brain injury, with $30 \%$ of players reporting at least one concussion in a season of club rugby. In a South African study ${ }^{35}$ concussion was reported to be the most prevalent injury in one season of high school Rugby Union making up $22 \%$ of all injuries, and another study ${ }^{45}$ documented a lifetime incidence of 2.3 concussions (range 0 - 7) per top team rugby-playing schoolboy. In each of these three studies, the researchers considered the figures to be an underestimate of the true concussion incidence in Rugby Union, due to athletes' common tendency to underreport, and the retrospective nature of the research. Finally, it would appear that injuries in Rugby Union at the professional level have been on the increase, and despite rule changes (New Zealand, Australia and Britain in the 1980s, and South Africa in 1990), this trend has shown no sign of abating. ${ }^{23}$

Moreover, in addition to the number of formally reported concussions, it is suggested that athletes are likely to sustain multiple subconcussive insults due to player-to-player and player-to-ground collisions over long periods of exposure to these sports, that are likely to have cumulative effects. ${ }^{24}$ Subconcussive injury (microtrauma) refers to events similar to those giving rise to concussion, but involving smaller impact forces that operate below the threshold necessary to produce symptoms. In light of this phenomenon there is also growing concern about the extent to which heading in soccer is a source of cumulative microtrauma to the brain, an issue about which there is uncertainty and ongoing debate. ${ }^{41}$

In sum, the figures from the studies cited above attest to the likelihood of an overall incidence of concussion of some magnitude (given the vast number of participants in these sports). The implications of this require careful evaluation on both the individual and societal levels.

\section{Neuropsychological consequences of MTBI in con- tact sport}

In recent years there has been growing concern regarding the extent of neuropsychological (cognitive, emotional and behavioural) changes that are known to occur in association with MTBI, and their implications. More immediate acute se- quelae typically resolve within 3 months post-injury, and effects that persist for longer than this are viewed as relatively intractable (i.e. chronic). ${ }^{39}$ Sequelae of traumatic brain injury (TBI), including MTBI, are non-specific in nature, and may include a number of symptoms varying in degree of severity depending on differential features of the injury (seriousness and location of the injury, and whether in the acute or chronic phase post-injury), all in interaction with the neurological and psychiatric predisposition of the injured individual. Typically following MTBI, cognitive effects identified on the basis of objective testing include dysfunction in memory, learning and processing speed, implicating fronto-temporal involvement. ${ }^{26}$ Emotional and behavioural effects described are headache, dizziness, blurred vision, anxiety, depression, sleep disturbance, noise and light sensitivity, fatigue, and a cluster of symptoms that implicate frontal lobe involvement, including distractibility, impulsivity, disinhibition, argumentativeness, irritability, aggression, impaired judgment, general psychosocial dysfunction, executive dysfunction, and lowered selfmotivation. $^{16,26,39}$

In both the acute and chronic conditions such sequelae may be marked, or they may be subtle yet perniciously present, only becoming apparent under stressful conditions or increased task challenge. ${ }^{12}$ A model of cognitive reserve has been proposed by researchers to account for the inconsistencies and variability amongst individuals who sustain TBls with similar levels of severity but where the outcome is different. ${ }^{43}$ In terms of this model, individuals who are most vulnerable to the deleterious effects of MTBI will be those who start off with reduced cerebral capacity due to factors such as a prior learning disability, low $I Q$, prior psychiatric disorder, and prior neurological damage including a prior head injury. Accordingly, research reveals that the majority of individuals will not reveal any clinically detectable permanent disability following a single MBTI. ${ }^{39}$

However, it is generally accepted that a substantial proportion of around $10-30 \%$ of such individuals do sustain chronic disability, particularly those with prior vulnerability such as cognitive or psychiatric disability, a factor that MTBI researchers increasingly urge should not be downplayed or overlooked. $^{39,40}$ These researchers emphasise the potential for type II error in group MTBI research (false-negatives), due to the confounding element of a substantial proportion of spared individuals in the cohort who serve to obscure the deleterious clinical picture for the relatively few who are significantly affected. Further, in keeping with cognitive reserve theory, it was already apparent from an early seminal study that individuals with the additive effect of cumulative mild brain injury do reveal detectable neuropsychological disability on a long-term basis. ${ }^{17}$ In support of this there has been a gathering weight of research across the spectrum of contact sports that points to permanent neurocognitive deficits demonstrated on objective testing, or symptomatic dysfunction based on self-reports, in players of these sports including soccer ${ }^{50}$, Australian Rules football, ${ }^{10}$ American football $^{22}$, and Rugby Union. ${ }^{45}$ Consistently, reviews point to problems being more pronounced in professional and 
older players with longer and/or more intensive exposure to the sport. ${ }^{2,45}$ Enhanced deficits in these older athletes is commensurate with cognitive reserve threshold theory, where the additive effect of repeated brain insults over a longer period of time has the effect of increasing vulnerability to symptom presentation.

In addition to length of exposure, it is of note that genetic vulnerability plays a role in the development of chronic traumatic brain damage. Research indicates that cognitive decline is more pronounced in older football players who carry the apolipoprotein e4 allele genotype. ${ }^{25}$ Commensurate with the cognitive reserve threshold model, ${ }^{43}$ this study highlights the potential for significant individual variability in symptom presentation that may occur in additive fashion, depending on the presence or absence of this genetic factor, as well as the variety of other risk factors described above, including the presence of prior MTBI in itself.

\section{Methodological evaluation of sports MTBI re- search}

For the most part sports MTBI studies that have compared sports groups with non-contact sports controls, have provided affirmation for the presence of deleterious effects in the target contact sport group. However, methodological criticism has been levied at some studies which have indicated that heading may be associated with deleterious effects, with the implication that outcome from these studies is only cautiously indicative of such effects. ${ }^{41}$ In particular, criticism has been raised around the frequently cited soccer studies of Matser and colleagues, ${ }^{30,31}$ on the grounds of alcohol use being a potentially confounding factor in the soccer group, and the use of multiple measures without making the appropriate adjustments for type I error (finding significance purely by chance). However, a response to this criticism indicates that the issue raised in respect of alcohol was not justified, and an adjustment for type I error was made in Matser's second 1999 study by implementing more stringent significance levels. ${ }^{31,32}$ Further, a number of significant strengths were in evidence for this study, including strict control for level of education and the inclusion of sports-active controls. A number of other studies that point to neuropsychological compromise in the contact sports groups ${ }^{41,45}$ similarly demonstrate methodological strengths, including control for education, IQ and gender, the incorporation of a sports-active control group, and the inclusion of overall incidence of concussion as a variable.

In contrast to multiple studies attesting to problems attributable to MTBI in contact sports groups (exemplified by those isolated for citation above), the present authors identified only 4 studies of comparative sports groups that did not support such deficits. ${ }^{3,20,38,48}$ However, all of these studies demonstrate substantive methodological limitations, and therefore do not provide convincing counterbalancing evidence to refute the presence of deleterious effects affirmed in the other studies. For example, two of these studies had no control for $I Q$, but in addition they used mixed male and female participants in their studies, constituting a serious confounding factor ${ }^{3,20}$ (see discussion below on differential gender effects). The other two studies had very small subgroup numbers (mainly in the low to mid 20s) and were therefore prone to type II error (failing to find significance when it does exist). ${ }^{38,48}$ Moreover one of these studies ${ }^{48}$ was on very young athletes (aged 13 - 16 years), and in terms of the cognitive reserve threshold model, any problems in this cohort could be expected to be very subtle and in some cases subclinical.

Several of the studies cited above that are in support of deleterious effects due to participation in a contact sport, also have small subgroup sample numbers. However, small cohorts are mainly a limiting factor for studies that fail to demonstrate significant differences, due to the associated loss of statistical power that may result in failure of an investigation to reveal meaningful changes. In contrast, relatively well-controlled studies that demonstrate statistically significant differences in the hypothesised direction, in spite of small sample numbers, gain potency with the implication that larger sample numbers would be likely to reveal even more marked effects.

Studies that have investigated neuropsychological effects within a sports cohort in terms of number of reported concussions (rather than contact versus non-contact groups), also predominantly demonstrate long-term cognitive or symptomatic deficit in association with increasing numbers of concussions, ${ }^{22,24}$ although there are a number of studies that do not do so. ${ }^{21,28}$ On examination, however, all those studies with negative findings reveal methodological problems in that usually there is limited control for age, education, IQ or gender, or all four of these potentially confounding variables. Furthermore, no concussion-based studies with negative findings identified by the authors had any non-contact sport-control group. Consequently all have the inherent confounding variable of unreported concussive or subconcussive episodes that may be present in the socalled zero concussion subgroups. This makes these studies particularly susceptible to type II error, and in order to establish differences they would need to have provided more strongly contrasting concussion groups. Accordingly, deleterious effects are supported on subgroups with $3+$ concussions, ${ }^{22}$ whereas those with negative findings have tended to investigate subgroups with a ceiling of only 1 or 2 concussions. $^{21,28}$

It is problematic that relatively isolated findings of negative effects with methodological problems such as outlined above, have been used to suggest that players of the targeted contact sports groups are safe from deleterious effects of multiple concussion. ${ }^{20,28}$ Findings such as these, which go against hypothetical expectations on theoretical and empirical grounds, need to be scrutinised rigorously for risk of type II error (false-negative effects), before making premature suggestions in respect of falsification of the expected effects. ${ }^{46}$ Another interpretive error that has occurred in the literature, has been to cite results of studies on post-injury follow-up of concussed players demonstrating return to baseline levels 
on cognitive tests, in order to suggest that athletes do not sustain persistent effects. ${ }^{33}$ However, in the absence of noncontact sports controls, return to baseline does not rule out the possibility that such athletes were impaired prior to the latest concussion under investigation, and/or are benefiting from practice effects.

\section{Gender issues}

Of the many studies cited above that implicate persistent deleterious consequences in association with participation in a contact sport, most pertain to male athletes. Two studies that have performed gender analyses on their sports cohorts provide support for the well-known fact that females and males perform differently on a number neurocognitive tests. ${ }^{3,20}$ Typically in terms of the literature ${ }^{26}$ females outperformed males on tests of visuoperceptual functioning ${ }^{3}$ and there were mixed results in terms of gender superiority for memory. ${ }^{3,20}$ Two studies were identified that examined neurocognitive effects within exclusively female sports groups. ${ }^{13,38}$ One study yielded negative findings in relation to heading in soccer for both males and females. ${ }^{38}$ As already indicated above, this study comprised small sample numbers thus being prone to type II error, therefore not ruling out the presence of heading effects for either group. The other study demonstrated visuoperceptual processing speed difficulties for female rugby players with concussions, ${ }^{13}$ suggesting that the established female advantage in this area did not protect these athletes from acquired decrements in association with rugby-related MTBI.

It appears that only one sports MTBI study has investigated symptomatic outcome with stratification for gender. ${ }^{1}$ This study demonstrated that headache was present for both males and females, whereas females reported more symptoms of being dazed and dizzy and males more symptoms of blurred vision, numbness and tingling. In a study on persistent symptomatic outcome following MTBI in general, female vulnerability to late complaints was demonstrated, supporting findings of prior research, and was considered to have a possible organic component. ${ }^{7}$ Overall the gender indications from this review are that females have a lower risk of concussive injury in sport than males. Furthermore, neurocognitive and symptomatic profiles differ for females compared with males, both in sports and more general contexts, and it appears that females may be at greater risk than males for persistent symptomatic problems post MTBI in general, of probable organic aetiology.

Of relevance here, also within the context of cognitive reserve threshold theory, is the common finding that female gender is a risk factor for Alzheimer's disease. ${ }^{43}$ Head injury is also a well-substantiated risk factor for Alzheimer's disease. ${ }^{18}$ Thus an implication from the cognitive reserve model is that when sports-related repetitive head injury is superimposed on the female brain with inherent pre-existing vulnerability to Alzheimer's disease, in additive fashion, this might further enhance the risk of a such a dementia in the female population. On the other hand, a counterbalancing protective factor might be that women play less intensively or collide at lower velocity than their heavier male counterparts, thus sustaining less severe effects. This supposition is supported by the literature cited above that confirms female concussion rates for elite-level participation in contact sports to be around $50 \%$ less than for males. ${ }^{8}$ Accordingly, in view of the differential empirical findings for gender (for rate of injury, neurocognitive test performance, symptomatic outcome and vulnerability factors), it is essential that research studies on the effects of concussion in contact sports not be investigated in mixed gender groups; rather there should be strict stratification for gender.

\section{Youth and scholastic issues}

Concern is being expressed about more immediate as well as long-term consequences of sports concussion that may easily go unacknowledged, and yet may translate into scholastic difficulties for the child, adolescent or university student. ${ }^{34}$ Cognitively such consequences include impairments in concentration, learning, and the ability to process information at speed which are known to occur after a MTBI for any period from days up to around 3 months. ${ }^{14,26}$ Examination dates at school and university may easily overlap with the continued presence of even quite short-term cognitive dysfunction in a sport-playing youth, and could make the difference between passing and failing in the borderline candidate, or the loss of a crucial scholarship for a bright scholar. It has been demonstrated that such effects may be perniciously subtle and out of the conscious awareness of the mildly concussed athlete albeit in evidence on objective testing, ${ }^{27}$ and hence the more immediate post-concussion effects (within days and weeks of the injury) need to managed with due caution in terms of possible deleterious effects on an athlete's scholarly activities.

Of relevance for youth-level participation in these sports, is that university football players with a history of two or more concussions and a learning disability reveal significantly worse performance on tests of executive function and speed of mental processing than those with a similar history of concussions and the absence of a learning disability. ${ }^{9} \mathrm{~A}$ recent study provides compelling evidence for persistent neurocognitive difficulties in a sample of symptom-free high school athletes with two or more concussions. ${ }^{34}$ This group was characterised by lower cumulative academic grade averages, and it is uncertain whether this was as a result of the concussion history, or a characteristic of those predisposed to concussion, or a combination of both of these factors. Some researchers have emphasised that the identified cognitive deficits following sports MTBI are subtle and have not as yet been shown to be of consequence in the players' everyday lives. ${ }^{20}$ Others emphasise how important it is that information on the cumulative and long-term effects of brain injury be communicated to young sports individuals, since a proportion of these individuals will have sustained what could be a permanent reduction in brain function prior to reaching maturity. ${ }^{15}$ 


\section{Societal implications}

Wide participation in contact sport at both youth and adult levels translates into very large numbers of individuals exposed to MTBI generally, and calls for an evaluation of possible consequences that go beyond the individual to the societal level.

In particular, the possibility of increasing violence as a result of structural brain damage incurred through large-scale participation in cerebrally hazardous contact sports appears to have been overlooked, although this is an element that has been examined in relation to TBI in general. One-year estimates of irritability and temper following severe TBI reportedly range from $30 \%$ to $70 \%$, and following MTBI from $5 \%$ to $70 \%$. ${ }^{47}$ One MTBI study showed that of those who did not suffer loss of consciousness, $21 \%$ presented with irritability. ${ }^{47}$ Accordingly 'aggression on little or no provocation' is proposed as a research criterion for post-concussive syndrome in the Diagnostic and Statistical Manual (DSMIV). ${ }^{37}$ Furthermore, it has been demonstrated that TBI in childhood may result in pervasive deficits in self-regulation, social and behavioural functioning, ${ }^{16}$ and frontal lobe damage in early life contributes to disability in the areas of insight, foresight, social judgement and empathy. ${ }^{36}$ It appears from research that a risk factor for the development of aggressive behaviours may include a pre-injury history of irritability and aggression. ${ }^{47}$ There is a high incidence of brain abnormality and/or a history of head injury amongst prisoners and people displaying antisocial behaviours, and a review of aggressive disorders reports that a small study of death-row inmates found a $75 \%$ history of TBI. ${ }^{47}$

Clearly, acquired brain damage identified in association with criminals and antisocial individuals would be the result of many causes, especially assault and motor vehicle accidents, and the intention here is not to suggest that sports participation is a predominant contributing factor to crime in our society. However, it is conceivable that for some people involved in cerebrally hazardous sports this may constitute a contributory effect. For example, it is probable that there will be an exacerbation of prior damage (from causes other than sport) in a proportion of individuals, through repeated MTBI sustained whilst participating in contact sports. Furthermore, those athletes with more aggressive dispositions are the ones most likely to be drawn to participate in these relatively aggressive sports. In light of the research cited above on risk factors for aggressive behaviour following $\mathrm{TBI},{ }^{47}$ these same individuals would constitute a subset susceptible to exacerbated aggressive tendencies, in association with damage to frontal systems that typically accompanies the concussive MTBI. Preliminary support has been gained for this supposition from studies on Rugby Union, where symptoms of 'argumentativeness' and 'aggression' differentiated rugby groups from non-contact sport controls more robustly than any other symptom. ${ }^{45}$ This may be a reflection of the rugby players being constitutionally more aggressive than the non-contact sports controls. However, the pervasiveness of this effect implies that there is an overlay of acquired argumentative/ aggressive traits due to repeated concussive incidents for a proportion of the rugby players in these studies.

In sum, on a societal level it would appear logical to expect increased aggressive tendencies in association with repetitive sports $\mathrm{MTBI}$ in a proportion of athletes, and this may be a silent contributing factor to antisocial behaviour and criminal activity in society. Further, exposure to repeated concussion has the potential to diminish brain power amongst the sector of our talented and not-so-talented youth who participate in these sports. Finally, due to increased risk of Alzheimer's disease following head injury, the extent to which sports-related concussion may contribute to increased risk in this regard especially for females is a matter of concern, and in need of further research. Epidemiological and longitudinal case-based studies are needed to substantiate the role of cerebrally hazardous sports in the development of the deleterious cognitive and behavioural changes described here, that may impact on an individual's scholastic or social life, or increase susceptibility to cognitive deterioration with aging.

\section{Conclusion}

In conclusion, the neuropsychological perspective presented here reveals concerns for deleterious effects on athletes in association with participation in contact sports, in the face of which health professionals can no longer afford to sit on the fence. There is growing evidence from research to support persistent problems in association with cumulative MTBI in association with the rugby football sports, at youth and adult levels. Studies identified that are in opposition to this are in the minority, and have methodological limitations including a high risk for type II error (false-negative results) that preclude any firm interpretations about the field contact sports being safe.

In this situation, movement in the direction of the following actions would appear appropriate: (i) participation in the contact sports should occur only after properly informed consent has been given, especially in the case of children and adolescents, thereby providing an educative function and medico-legal protection to the sports body involved; (ii) comprehensive monitoring of every sports concussion case on an individualised basis should be implemented according to the consensus opinion arising out of the recent concussion management symposia, that discredits generalised management guidelines based on relatively arbitrary cut-off criteria; ${ }^{19}$ (iii) neuropsychological assessment should be included as a crucial component of the overall management programme, incorporating computer-based pre-and post-concussion neurocognitive screening ${ }^{19}$ (iv) such neurocognitive screening should be mandatory in schools that promote rugby due to a particularly high risk of concussion in the sport; $(v)$ individualised return-to-play decisions, and advice on termination of participation in a contact sport, should be made with particular caution around vulnerability factors for deleterious outcome, such as prior concussions, cognitive dysfunction, psychiatric and neurological disability, 
and persistent aggressive or antisocial tendencies where there is little or no provocation; and (vi) in cases where a decision on termination is proving to be particularly difficult for the athlete, and given available financial resources, consideration might even be given to investigation for the presence of the apolipoprotein e4 allele genotype.

For best practice in the implementation of such returnto-play and termination decisions using computerised psychometric instruments, registered psychologists with training in clinical neuropsychology are called for, and have an important complementary role to play. ${ }^{19,29}$ Specialist skills in psychometric test usage, brain-behaviour relations and psychiatric diagnosis, enables them to conduct a differentiated evaluation of an athlete's post-concussive cognitive, emotional and behavioural status. The ease with which the computer-based test profiles can be transmitted by e-mail greatly facilitates the doctor-psychologist liaison necessary for widespread sports concussion services to occur at the highest professional level in a relatively cost-effective manner. Nevertheless, substantial healthrelated funding will be needed to implement optimal concussion management amongst sports populations that are economically disadvantaged, such as pertains to large numbers of individuals in a country such as South Africa.

\section{Acknowledgements}

Research for this review was funded by the National Research Foundation (NRF) and the Rhodes University Council.

Incidence figures are rounded to the nearest decimal point. Due to space restrictions citations were kept to a minimum. An alternative version of this article with a comprehensive set of references is available on request from the corresponding author.

The authors are commercially involved in the development of neurocognitive screening as a component of sports concussion management in South Africa and Britain, using the ImPACT programme.

\section{REFERENCES}

1. Barnes BC, Cooper L, Kirkendall DT, McDermott TP, Jordan BD, Garrett WE. Concussion history in elite male and female soccer players. Am J Sports Med 1998; 26: 433-8.

2. Baroff GS. Is heading a soccer ball injurious to brain function? J Head Trauma Rehabil 1998; 13: 45-52.

3. Barr WB. Neuropsychological testing of high school athletes. Arch Clin Neuropsychol 2003; 18: 91-101.

4. Bathgate A, Best JP, Craig G, Jamieson M. A prospective study of injuries to elite Australian rugby union players. Br J Sports Med 2002; 36: 265-9.

5. Behr M. Beyond the grass ceiling. Fairlady, October 2001: 49-51.

6. Bird YN, Waller AE, Marshall SW, Alsop JC, Chalmers DJ, Gerrard DF. The New Zealand rugby injury and performance project: V. Epidemiology of a season of rugby injury. Br J Sports Med 1998; 32: 319-25.

7. Bohnen N, van Zutphen W, Twijinstra A, Wijnen G, Bongers J, Jolles J. Late outcome of mild head injury: Results from a controlled postal survey. Brain Inj 1994; 8: 701-8.

8. Cassidy JD, Carrol LJ, Peloso PM, Borg J, von Holst H. Incidence, risk factors and prevention of mild traumatic brain injury: results of the WHO collaborating centre task force on mild traumatic brain injury. J Rehabil Med 2004; Suppl 43, 28-60.

9. Collins MW, Grindell SH, Lovell MR, et al. Relationship between concussion and neuropsychological performance in college football players. JAMA 1999; 282: 964-70.

10. Cremona-Meteyard SL, Geffen GM. Persistent visuospatial attention deficits following mild head injury in Australian rules football players. Neuropsychologist 1994; 32: 649-62.

11. Delaney JS, Lacroix VJ, Gagne C, Antoniou J. Concussions among university football and soccer players: A pilot study. Clin Sports Med 2001; 11: $234-40$.

12. Ewing R, McCarthy D, Gronwall D, Wrightson P. Persisting effects of minor head injury observable during hypoxic stress. J Clin Neuropsychol 1980; 2: $147-55$.

13. Farace E, Ferree RM, Hollier JA, Barth JT, Shaffrey ME. Trails A: Neurocognitive effect of previous concussions in woman's rugby sample (Abstract). J Int Neuropsychol Soc 2003; 9: 207.

14. Frencham KAR, Fox AM, Maybery MT. Neuropsychological studies of mild traumaic brain injury: a meta-analytic review of research since 1995. J Clin Exp Neuropsychol 2005; 27: 334-51.

15. Gaetz M, Goodman D, Weinberg H. Electrophysiological evidence for the cumulative effects of concussion. Brain Inj 2000; 14: 1077-88.

16. Ganesalingam K, Sanson A, Anderson V, Yeates KO. Self-regulation and social and behavioral functioning following childhood traumatic brain injury. J Int Neuropsychol Soc 2006; 12: 609-21.

17. Gronwall D, Wrightson P. Cumulative effect of concussion. Lancet 1975; 2: 995-7.

18. Guo Z, Cupples LA, Kurz A, et al. Head injury and the risk of AD in the MIRAGE study. American Academy of Neurology 2000; 54: 1316-23.

19. Guskiewicz KM, Bruce DE, Cantu RC, et al. National Athletic Trainers' Association position statement: Management of sport-related concussion. Journal of Athletic Training 2004; 39: 297.

20. Guskiewicz KM, Marshall SW, Broglio S, Cantu RC, Kirkendall DT. No evidence of impaired neurocognitive performance in collegiate soccer players. Am J Sports Med 2002; 30: 157-62.

21. Iverson GL, Brooks BL, Lovell MR, Collins MW. No cumulative effects for one or two previous concussions. Br J Sports Med 2006; 40: 72-5.

22. Iverson GL, Gaetz M, Lovell MR, Collins MW. Cumulative effects of concussion in amateur athletes. Brain Inj 2004; 18: 433-43.

23. Jakoet I, Noakes TD. A high rate of injury during the 1995 Rugby World Cup. S Afr Med J 1998; 88: 45-7.

24. Killam C, Cautin RL, Santucci AC. Assessing the enduring residual neuropsychological effects of head trauma in college athletes who participate in contact sports. Arch Clin Neuropsychol 2005; 20: 599-611.

25. Kutner KC, Erlanger DM, Tsai J, Jordan BD, Relkin NR. Lower cognitive performance of older football players possessing apolipoprotein $\mathrm{E}$ epsilon 4. Neurosurgery 2000; 47: 651-7.

26. Lezak MD, Howieson DB, Loring DW. Neuropsychological Assessment. 4th ed. Oxford: Oxford University Press, 2004.

27. Lovell MR, Collins MW, Iverson GL, Johnston KM, Bradley JP. Grade 1 or 'ding' concussions in high school athletes. J Neurosurg 2004; 98: 296301.

28. Maddocks DL, Saling M, Dicker GD. A note on normative data for a test sensitive to concussion in Australian Rules footballers. Australian Psychologist 1995; 30: 125-7.

29. Makgoke P. Media statement by the Health Professions Council of South Africa (HPCSA). South African Psychiatry Review 2004; 7: 39.

30. Matser JT, Kessels AG, Jordan BD, Lezak MD, Troost J. Chronic traumatic brain injury in professional soccer players. Neurology 1998; 51: 791-6.

31. Matser J, Kessels AG, Lezak MD, Jordan BD, Troost J. Neuropsychological impairment in amateur soccer players. JAMA 1999; 282: 971-3.

32. Matser E, Kessels A, Troost K, Lezak M. Letter in reply to Dr Babbs and Drs Grote and Donders. JAMA 2000; 16: 883.

33. McCrory P. When to retire after concussion? Br J Sports Med 2001; 35 : 379-82.

34. Moser RS, Schatz P, Jordan BD. Prolonged effects of concussion in high school athletes. Neurosurgery 2005; 17: 91-100.

35. Nathan M, Goedeke R, Noakes TD. The incidence and nature of rugby 
injuries experienced at one school during the 1982 rugby season. S Afr Med J 1983; 64: 132-7.

36. Price BH, Daffner KR, Stowe RM. The comportmental learning disabilities of early frontal lobe damage. Brain Inj 1990; 113: 1383-93.

37. American Psychiatric Association. Diagnostic and Statistical Manual of Mental Disorders. 4th ed. Text revision (DSM IV-TR). Washington, DC. APA, 2000.

38. Putukian M, Echemendia RJ, Mackin S. The acute neuropsychological effects of heading in soccer: a pilot study. Clin J Sport Med 2000; 10: 104-

39. Reitan RM, Wolfson D. The two faces of mild head injury. Arch Clin Neuropsychol 1999; 14: 191-202.

40. Ruff R. Two decades of advances in understanding of mild traumatic brain injury. J Head Trauma Rehabil 2005; 20: 5-18.

41. Rutherford A, Stephens R, Potter D. The neuropsychology of heading and head trauma in association football (soccer): A review. Neuropsychol Rev 2003; 13: 153-79.

42. South African Press Association. Legal now for women to go boxing. Eastern Province Herald, 24 August 2001: 4.

43. Satz P. Brain reserve capacity on symptom onset after brain injury: A for- mulation and review of evidence for threshold theory. Neuropsychology 1993; $7:$ :273-95

44. Seward $\mathrm{H}$, Orchard J, Hazard $\mathrm{H}$, Collinson D. Football injuries in Australia at the elite level. Med J Aust 1993; 159: 298-301.

45. Shuttleworth-Edwards AB, Border M, Reid I, Radloff S. South African Rugby Union. In: Lovell MR, Echemendia RE, Barth JT, Collins MW, eds. Traumatic Brain Injury in Sports: An International Neuropsychological Perspective. Lisse, The Netherlands: Swets and Zeitlinger, 2004: 149-68.

46. Shuttleworth-Jordan AB. Age and education effects on brain-damaged subjects: negative findings revisited. Clin Neuropsychol 1997; 11: 205-9.

47. Silver JM, Yudofsky SC, Anderson KE. Aggressive disorders. In: Silver JM, McAllister TM, Yudofsky SC, eds. Textbook of Traumatic Brain Injury. Washington, DC: American Psychiatric Publishing, 2005: 259-77.

48. Stephens R, Rutherford A, Potter D, Fernie G. Neuropsychological impairment as a consequence of football (soccer) play and football heading: a preliminary analysis and report on school students (13-16 years). Child Neuropsychology 2005; 11: 513-26.

49. Wills SM, Leathem JM. An investigation of brain injury incurred in New Zealand club-grade rugby. J Int Neuropsychol Soc 2001; 7: 405.

50. Witol AD, Webbe FM. Soccer heading frequency predicts neuropsychological deficits. Arch Clin Neuropsychol 2003; 18: 417.

\section{3. 8. sosing

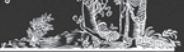 ELSEVIER \\ WHAT IS AN @)dition? \\ An $\Theta$ dition is a revolutionary new format that gives you both the updated version of the book, and also a state-of-the-art website. As well as allowing you to consult the entire contents of the book online, each website includes continuous content updates, fully searchable text, easily downloadable electronic images, and much more!}

\section{Elsevier's Fantastic edition promotion}

\section{HOW DOES THE PROMOTION WORK?}

Featuring 8 of Elsevier's major and established e-dition reference works, this offer allows you to have a 28 day FREE trial of a state-of-the-art website plus an exclusive $10 \%$ discount when you purchase an $\Theta$ dition from THIS store.

\section{HOW DO I TAKE PART?}

Taking part is easy! All you have to do is contact the below email address, state which of the 8 books (depending on if it's published!) you would like to trial, and we will send you a FREE 28 day PIN code. In addition to this, when you return to the bookstore to purchase the book, simply show this card and receive a fantastic $10 \%$ discount!

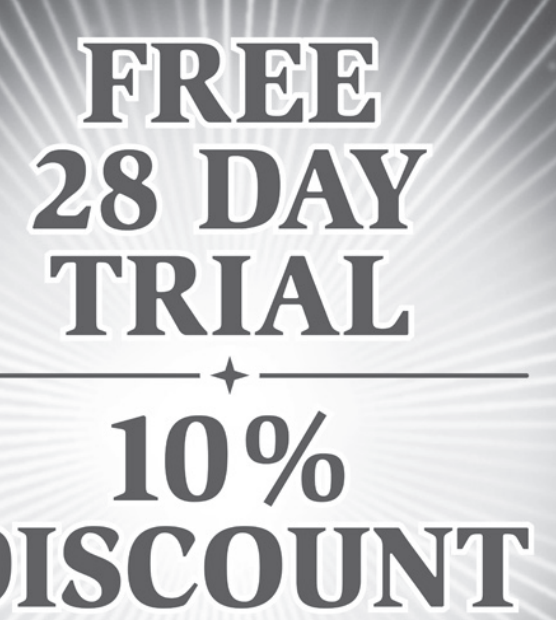

Running across 8 titles throughout the year!

\section{Simply email 'edition trial' to brents@hmpg.co.za to arrange your pin!}

